\title{
Performance Analysis of Coded Cooperation based Relayed Transmission under Rayleigh Fading Channels
}

\author{
Asaduzzaman*, Hyung Yun Kong* ${ }^{\circ}$ Regular Members
}

\begin{abstract}
This paper introduces the coded cooperation protocol in wireless relay network. We propose a two relay based coded cooperation protocol with RCPC codes for wireless networks. The proposed two-relay based system can achieve a diversity of order 4 under slow fading environment. Under fast fading, the diversity order is 2 times of the free distance of the convolutional code. We develop upper bounds on BER and FER for the system under both slow and fast fading with Rayleigh distribution. The effect of various channel conditions on the cooperation is also examined in this work.
\end{abstract}

Key Words : Coded cooperation, Rayleigh Fading, Coding gain, Diversity gain, Relay network

\section{Introduction}

In order to meet the demands of multimedia communications, next-generation wireless systems must employ advanced algorithmsand techniques that not only increase the data rate, but also enable the system to guarantee the quality of service (QoS) desired by the various media classes. Wireless channels are vulnerable to fading effect. To combat against fading, among with other techniques diversity is of primary importance because of the random nature of the wireless environment. Space time block coding using multiple transmit antenna opens a new paradigm of communication over fading channels. However, many wireless devices are limited by size or hardware complexity to one antenna due to size or other constraints ${ }^{[1]}$, therefore, the conventional space-time coding cannot be used for transmit diversity. To overcome this restriction, a new technique, called cooperative transmission was born that allows single antenna mobiles to gain some benefits of spatial diversity. These advantages are practically achievable by using a collection of distributed antennas belonging to multiple users as a virtual antenna array. There are three basic cooperative communications protocols: amplify-andforward, decode-and-forward and Coded cooperation ${ }^{[1]}$.

This paper proposes a novel protocol to implement the idea of coded cooperation in wireless relay system where one active user is assisted by a number of relays. Coded cooperation diversity is an active user cooperation technique where cooperative signaling has been integrated with channel coding was introduced by Todd E. Hunter and Aria Nosratinia in [2]. The main idea of this proposal was users share their antennas such that a portion of each user's code bits arrive at the base station through a different and independent fading channel. Bit error performance, outage behavior and various implementation issues of coded cooperation has been extensively studied in the literature ${ }^{[2-5\}}$.

We introduce the coded cooperation technique in wireless relay network where one or more idle users assist one active user like a relay. So far, amplify-and-forward (AF), decode-and-re-encode (DR) and decode-and-forward (DF)protocol for wireless relay network has been studied in various scenarios $^{[9-11]}$. To get the coding gain along with the

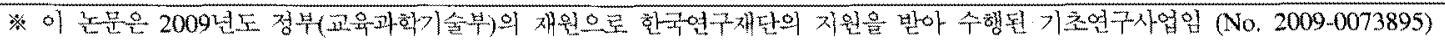

* 울산대학교 전기전자정보시스템공학후 무선통신 실혐실asad78@mail.ulsan.ac.kr, hkong@mail.ulsan.ac.kr), $\left({ }^{\circ}:\right.$ 고신저자)

혼문번호 : KICS2010-06-287, 접수일자 : 2010년 6월 29일, 최종논문접수일자: 2011년 4월 3일
} 
diversity gain cyclic code ${ }^{[7]}$ and LDPC $^{[8]}$ based decode and forward protocol has been also proposed. The convolutional codes are the most efficient and popular forward error correction (FEC) technique in flat, slow Rayleigh fading channels. Albeit, the complexity of Viterbi decoder is high but the recent development in hardware technology encourage us to investigate the coded cooperation in relay networks because, coded cooperation protocol benefited from coding gain along with diversity gain. In this paper we utilize the idle users in a wireless network as relay for the active users.

We consider the well known RCPC based two frame structure of coded cooperation applied in [2-5] as the basic structure of cooperation. The benefit of this kind of coding structure (coding gain) has been well studied in literature ${ }^{[17]}$. In this paper we emphasis our analysis on diversity gain in wireless relay network when coded transmission is used. We propose a coded cooperation scheme that utilizes the benefits of both MRC based cooperative protocol and coded cooperation protocol. We also develop the Upper Bound on BER and FER of this mixed combined signal for convolutional code. An active user divides the convolutional coded bits in two parts by means of puncturing ${ }^{[6]}$. In the first phase, active user transmits the first frame of the coded bits towards the destination and relays. Relays try to decode the received signal at first phase. In the second phase, active user transmits the second frame of the coded bits and two relays transmit first and second frame of the coded bits towards the destination. Active user transmits two frame in two coherent time slot of independent fading statistics so, a time diversity (incase of TDMA) is also obtained along with spatial diversity.

Our proposed protocol is applicable in cellular based wireless network as well as distributed wireless network where some redundant relay or idle users are available. The rest of the paper is organized as follows. Section 2 describes the system model for mobile user, relay and the destination. Our proposed protocol is explained in section 3. Performance analysis to develop bonds on BER and FER is done in section 4 . In section 5 we support our idea with some numerical simulation and finally we conclude this paper in section 6 .

\section{System Model}

Consider a cooperative transmission in a generic wireless network consisting of single antenna entities: mobile users and Destination (D) depicted in fig. 1. The mobile users operate in two different modes: Source (S) and Relay (R). All the mobile users are equipped with a RCPC encoder and Viterbi decoder. The source node encodes a block of information bits using CRC encoder so that the relay can detect whether the received frame is correct or not.

The CRC encoded bits are further encoded through a RCPC encoder as explained in [5]. We consider the total $\mathrm{N}$ encoded bits are divided in two blocks of $N_{1}$ and $N_{2}$. Here all $\mathrm{N}, N_{1}$ and $N_{2}$ are valid code, but $\mathrm{N}$ is more powerful code than $N_{1}$ and $N_{2}$. In relay mode, mobile users receive the $N_{1}$ code word from a source node and decode it with the Viterbi decoder. The decoded bits are re-encoded by RCPC encoder and transmit towards the destination on the basis of CRC result. If the relays can't decode the bits correctly (checked by CRC) it will be in silent mode otherwise, it will transmit corresponding block of data i.e. relay-1 will transmit $N_{1}$ and relay-2 will transmit $N_{2}$.

We assume mobile users has own orthogonal channel towards the destination and the system is free from inter-symbol interference. The channels (source to destination, source to relay and relay to

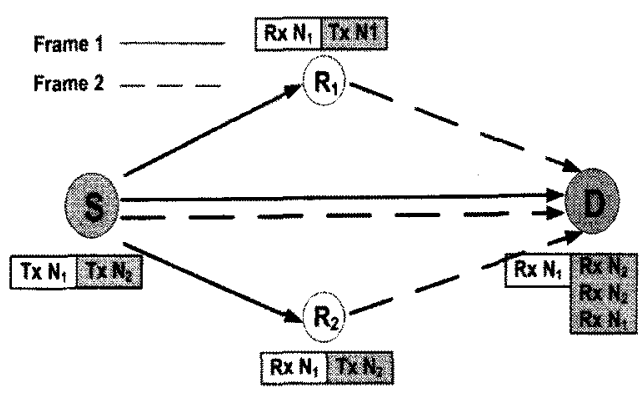

Fig. 1 System Model 
destination) are assume to be Rayleigh fading with plus Additive White Gaussian Noise (AWGN). The destination performs simple Maximal Ratio Combiner (MRC) algorithm to combine the same frame of coded signals from different antenna and then convolutional coded data bits are decode using Viterbi algorithm. We also assume the perfect channel state information is available at each receivers but not at the transmitters.

We consider the source transmits two frame of proposed coded frame structure in two independent channel coherent times i.e. they experience independent fading path. Therefore, in our proposal destination receives signal through four independent fading paths (two independent paths from source and two independent paths from two relays). Fig. 1 shows the signal received at destination in two different frames and makes this visible. So a diversity of order 4 is achieved. The basic idea of this paper which same system model was presented in [17]. In this paper we give a detail performance analysis of the proposed system.

\section{Proposed Protocol}

In the first phase the source node transmits $N_{1}$ bits towards the destination and the relays. The received signal at destination and the relays from source in phase- 1 is given by the equations

$$
\begin{aligned}
& r_{S 1}^{(1)}(n)=q_{S 1}^{(1)} \sqrt{P_{S}} a_{1}(n)+\eta_{S 1}^{(1)}(n) \\
& r_{S 2}^{(1)}(n)=q_{S 2}^{(1)} \sqrt{P_{S}} a_{1}(n)+\eta_{S 2}^{(1)}(n) \\
& r_{S D}^{(1)}(n)=q_{S D}^{(1)} \sqrt{P_{S}} a_{1}(n)+\eta_{S D}^{(1)}(n)
\end{aligned}
$$

where $r_{i j}^{(1)}(n)$ is signal received at node $\mathrm{j}$ from node $i$ in first phase. $\eta_{i j}^{(1)}(n)$ is the complex AWGN samples at node $\mathrm{j}$, in phase- 1 and modeled as independent across time and antenna with zero mean and variance $N_{0} / 2$ per dimension. The coefficient $q_{i j}^{(1)}$ is represent the quasi static flat fading (constant over one frame for each user and independent from user to user for slow fading case) coefficient between node $i$ and $j$ in phase-1 with Rayleigh distribution and modeled as zero mean complex Gaussian random variable with $\lambda_{i j}^{2} / 2$ per dimension. The signal $a_{1}(n)$ is the BPSK modulated symbols of $N_{1}$ bits $(\in\{+1,-1\})$ and $P_{s}$ is energy transmitted per bit by source. At the destination received $N_{1}$ is simply stored and two relays $\left(R_{1}, R_{2}\right)$ estimate the BPSK modulated signal $a_{1}(n)$ by making the phase correction as

$$
\begin{aligned}
& \widehat{a_{1 R_{1}}}(n)=R E\left(q_{S 1}^{(1)^{*}} r_{S 1}^{(1)}\right) \\
& \widehat{a_{1 R_{2}}}(n)=R E\left(q_{S 2}^{(1)^{*}} r_{S 2}^{(1)}\right)
\end{aligned}
$$

Where, $\widehat{a_{1 j}}(n)$ is the detected signal at node $\mathrm{j}$ and $\mathrm{x}^{*}$ represents complex conjugate of $\mathrm{x}$. Using Viterbi algorithm we can easily decode the estimated copy of $N_{1}$ to recover the actual information. Decoded information bits are checked by CRC to know whether the received bits are decoded correctly or not.

In second phase, each relay transmits the re-encoded bits conditionally. If the decoding is successful then relay-1 $\left(R_{1}\right)$ generates puncture $N_{1}$ bits and relay-2 $\left(R_{2}\right)$ generates puncture $N_{2}$ bits using the RCPC encoder. In this phase relay-1 transmits $N_{1}$ bits and relay-2 and source transmit $N_{2}$ bits towards the destination using their own orthogonal channels. Received data at the destination for second phase is given by

$$
\begin{aligned}
& r_{S D}^{(2)}(n)=q_{S D}^{(2)} \sqrt{P_{S}} a_{2}(n)+\eta_{S D}^{(2)}(n) \\
& r_{1 D}^{(2)}(n)=q_{1 D}^{(2)} \sqrt{P_{R}} a_{1}(n) \varepsilon_{1}+\eta_{1 D}^{(2)}(n) \\
& r_{2 D}^{(2)}(n)=q_{2 D}^{(2)} \sqrt{P_{R}} a_{2}(n) \varepsilon_{2}+\eta_{2 D}^{(2)}(n)
\end{aligned}
$$

where, the AWGN $\eta_{2, i j}(n)$ and fading coefficient $q_{2, i j}$ can be similarly defined as first phase. The signal $a_{2}(n) \in\{+1,-1\}$ is the BPSK modulated symbol of $N_{2}$ bits and $\operatorname{Pr}$ is the transmitted power from both the relay. $\varepsilon i$ in second and third equation represent the conditional transmission from the relays. If relay- $i$ can decode the information bits correctly $\varepsilon i=1$, else $\varepsilon i=0$.

The destination received two independent copies 
of $N_{1}$ and $N_{2}$ signal. These two copies are optimally combined using MRC ${ }^{[12]}$ and the estimated $a_{1}(n)$ and $a_{2}(n)$ can found as,

$$
\begin{aligned}
& a_{1}=\operatorname{Re}\left(\frac{q_{S D}^{(1)} \sqrt{P_{s}}}{\left(\sigma_{S D}^{(1)}\right)^{2}} r_{S D}^{(1)}+\varepsilon_{1} \frac{q_{1 D}^{(2) *} \sqrt{P_{r}}}{\left(\sigma_{1 D}^{(2)}\right)^{2}} r_{1 D}^{(2)}\right) \\
& a_{2}=\operatorname{Re}\left(\frac{q_{S D}^{(2)} \sqrt{P_{s}}}{\left(\sigma_{S D}^{(2)}\right)^{2}} r_{S D}^{(2)}+\varepsilon_{2} \frac{q_{1 D}^{(2) *} \sqrt{P_{r}}}{\left(\sigma_{2 D}^{(2)}\right)^{2}} r_{2 D}^{(2)}\right)
\end{aligned}
$$

If any relay fails to decode the data correctly ( $\varepsilon$ $i=0$ ) then detection algorithm in equation (4) becomes simple $\mathrm{ML}^{[12]}$ detection. The destination can recognize the silence mode of any relay by measuring the SNR at the second phase from the relays.

Now destination performs de-puncturing ${ }^{[6]}$ and after Viterbi decoding and eliminating the CRC bits we can get the actual transmitted signal from the source. The two branch MRC based spatial diversity combiner offer a diversity order of $2^{[10]}$ and the coded cooperation technique also provide a diversity order of $2^{[5]}$. In this work, we applied both the technique together so we expect diversity order of 4 . Simulation result and mathematical analysis justify this and show that the new protocol outperforms the performance of the direct transmission and single user time diversity or conventional coded cooperation.

\section{Performance Analysis}

pproaches to arriving Bit Error Rate (BER) and Frame Error Rate (FER) bounds, we employ the so-called pair wise error probability (PEP); the probability of choosing one symbol sequence over another for a given pair of possible transmitted symbol sequences. BER and FER can obtain by performing a weighted summation over all pair wise events.

\section{1. pair wise Error Probability}

The probability of choosing the coded sequence $c^{\prime}=\left(c_{1}, c_{2}, \ldots \ldots, c_{n}\right)$ when $c=\left(c_{1}, c_{2}, \ldots \ldots ., c_{n}\right)$ was transmitted given that these are the only two possible choices. The PEP for convolutionally encoded BPSK conditioned on fading coefficients $q=\left(q_{1}, q_{2}, \ldots, q_{n}\right)$ can be written as [12],

$$
P(c \rightarrow c \mid q)=Q\left(\sqrt{2 \sum} \gamma(N)\right)
$$

where, the set $\eta$ is the set of all $\mathbf{n}$ for which $c_{n} \neq \hat{c_{n}}$ and $Q(x)$ Gaussian $Q$-function is $\gamma(n)$ is instantaneous signal to noise ratio (SNR) of the received bit $\mathrm{n}$, and defined as

$$
\gamma(n)=\frac{P_{n}}{N_{0, n}}|q(n)|^{2}
$$

\subsubsection{Slow Fading case:}

In this case fading coefficients for each channel are constant over the channel coherent time i.e. $q(n)=q$ and $\gamma(n)=\gamma$ for all $n$. For linear codes the PEP depend on the Hamming distance $\mathrm{d}$ between $c_{n}$ and $\hat{c_{n}}$ not on code words. So the conditional PEP can be denoted as

$$
P(d \mid \gamma)=Q(\sqrt{2 d \gamma})
$$

The instantaneous received SNR for first frame of coded bit $N_{1}$ at destination can found from equation (3) and (4) as

$$
\gamma_{1}=\frac{P_{s}\left|q_{1, S D}\right|^{2}}{N_{0}}+\varepsilon_{1} \frac{P_{R}\left|q_{1 D}\right|^{2}}{N_{0}}=\gamma_{1 a}+\varepsilon_{1} \gamma_{1 b}
$$

where, $\gamma_{1 a}$ and $\gamma_{1 b}$ denote the SNR of two MRC branch related to $\gamma_{1}$. Similarly for $N_{2}$ we can write,

$$
\gamma_{2}=\frac{P_{s} \mid q_{2 . S D}{ }^{2}}{N_{0}}+\varepsilon_{2} \frac{P_{R}\left|q_{2 D}\right|^{2}}{N_{0}}=\gamma_{2 a}+\varepsilon_{2} \gamma_{2 b}
$$

Total code word $\mathrm{N}$ is recombination of $N_{1}$ and $N_{2}$ according to the puncturing table. So we can assume that the hamming distance between transmitted and received code words $\left(c_{n}\right.$ and $\hat{c_{n}}$ ) are divided as $d=d_{1}+d_{2}$, where 


$$
\frac{d_{1}}{d_{2}}=\frac{N_{1}}{N_{2}}
$$

Now using equation (8), (9) and (10) we can rewrite (7) as

$$
\begin{aligned}
& P\left(d \mid \gamma_{1 a}, \gamma_{1 b}, \gamma_{2 a}, \gamma_{2 b}\right) \\
& =Q\left(\sqrt{2 d_{1} \gamma_{1 a}+2 d_{1} \varepsilon_{1} \gamma_{1 b}+2 d_{2} \gamma_{2 a}+2 d_{2} \varepsilon_{2} \gamma_{2 b}}\right)
\end{aligned}
$$

We can get the unconditional PEP over the pdf by averaging the equation (11) as,

$$
\begin{array}{r}
P(d)=\int_{0}^{\infty} \int_{0}^{\infty} \int_{0}^{\infty} \int_{0}^{\infty} P\left(d \mid \gamma_{1 a}, \gamma_{1 b}, \gamma_{2 a}, \gamma_{2 b}\right) \\
P\left(\gamma_{1 a}\right) P\left(\gamma_{1 b}\right) \times P\left(\gamma_{2 a}\right) P\left(\gamma_{2 b}\right) d \gamma_{1 a} \gamma_{1 b} \gamma_{2 a} \gamma_{2 b}
\end{array}
$$

where, $p(x)$ is probability density function (pdf) of $x$. The integration of equation (12) can be calculated using the well known alternative form of Gaussain $Q$-function developed by Craig $^{[14]}$.

$$
Q(x)=\frac{1}{x} \int_{0}^{\Pi / 2} \exp \left(-\frac{x^{2}}{2 \sin ^{2} \theta}\right) d \theta, x \geq 0
$$

Using (11) and (12) and (13),

$$
\begin{aligned}
P(d) & = \\
& \frac{1}{\pi} \int_{0}^{\pi / 2}\left[\int_{0}^{\infty} \exp \left(-\frac{d_{1} \gamma_{1 a}}{\sin ^{2} \theta}\right) P\left(\gamma_{1 b}\right) d \gamma_{1 a}\right] \\
& \times\left[\int_{0}^{\infty} \exp \left(-\frac{\varepsilon_{1} d_{1} \gamma_{1 b}}{\sin ^{2} \theta}\right) P\left(\gamma_{1 b}\right) d \gamma_{1 b}\right] \\
\times & {\left[\int_{0}^{\infty} \exp \left(-\frac{d_{2} \gamma_{2 a}}{\sin ^{2} \theta}\right) P\left(\gamma_{2 a}\right) d \gamma_{2 a}\right] } \\
\times & {\left[\int_{0}^{\infty} \exp \left(-\frac{\varepsilon_{2} d_{2} \gamma_{2 b}}{\sin ^{2} \theta}\right) P\left(\gamma_{2 b}\right) d \gamma_{2 b}\right] d \theta }
\end{aligned}
$$

Following the technique of Simon and Alouini ${ }^{[13]}$ we can find the exact solution of (14) for Rayleigh distribution as,

$$
\begin{aligned}
P(d)= & \frac{1}{\pi} \int_{0}^{\pi / 2}\left(1+\frac{d_{1} \overline{\gamma_{1 a}}}{\sin ^{2} \theta}\right)^{-1}\left(1+\varepsilon_{1} \frac{d_{1} \overline{\gamma_{1 b}}}{\sin ^{2} \theta}\right)^{-1} \\
& \left(1+\frac{d_{2} \overline{\gamma_{2 a}}}{\sin ^{2} \theta}\right)^{-1}\left(1+\varepsilon_{2} \frac{d_{2} \overline{\gamma_{2 b}}}{\sin ^{2} \theta}\right)^{-1} d \theta
\end{aligned}
$$

where $\bar{\gamma}$ represents the average SNR of the corresponding links. Equation (15) is the exact expression of the unconditional PEP and can easily be evaluated with numerical integration technique. We can obtain the upper bound by setting $\sin ^{2} \theta=1$ and performing the integration of (15) as

$$
P(d) \leq \frac{1}{2}\left(\begin{array}{l}
\left.\frac{1}{1+d_{1} \overline{\gamma_{1 a}}}\right)\left(\frac{1}{\frac{1}{1+d_{1} \varepsilon_{1} \overline{\gamma_{1 b}}}}\right)\left(\frac{1}{1+d_{2} \overline{\gamma_{2 a}}}\right) \\
\frac{1}{1+d_{2} \varepsilon_{2} \bar{\gamma}_{2 b}}
\end{array}\right)
$$

For two relay assisting one active user we have to consider the following 3 cases. Case-1: both relays successfully decode the information $\left(\varepsilon_{1}=\varepsilon_{2}\right.$ $=1$ ). In this case the two portions of the coded bits ( $N_{1}$ and $N_{2}$ ) arrived through different channels. $N_{1}$ is transmitted by source and relay- 1 . Conditional PEP can found from (11) by replacing the values of $\mathcal{E}_{1}$ and $\varepsilon_{2}$

$$
\begin{aligned}
& P\left(d \mid \gamma_{1 a}, \gamma_{1 b}, \gamma_{2 a}, \gamma_{2 b}\right) \\
& =Q\left(\sqrt{2 d_{1} \gamma_{1 a}+2 d_{1} \gamma_{1 b}+2 d_{2} \gamma_{2 a}+2 d_{2} \gamma_{2 b}}\right)
\end{aligned}
$$

We can obtain the unconditional exact PEP and upper bound from (15) and (16) as,

$$
\begin{gathered}
P(d)=\frac{1}{\pi} \int_{0}^{\pi / 2}\left(1+\frac{d_{1} \overline{\gamma_{1 a}}}{\sin ^{2} \theta}\right)^{-1}\left(1+\frac{d_{1} \overline{\gamma_{1 b}}}{\sin ^{2} \theta}\right)^{-1} \\
\left.1+\frac{d_{2} \overline{\gamma_{2 a}}}{\sin ^{2} \theta}\right)^{-1}\left(1+\frac{d_{2} \overline{\gamma_{2 b}}}{\sin ^{2} \theta}\right)^{-1} d \theta \\
P(d) \leq \frac{1}{2}\left(\frac{1}{1+d_{1} \overline{\gamma_{k a}}}\right)\left(\frac{1}{1+d_{1} \overline{\gamma_{1 b}}}\right) \\
\left(\frac{1}{1+d_{2} \overline{\gamma_{2 a}}}\right)\left(\frac{1}{1+d_{2} \bar{\gamma}_{2 b}}\right)
\end{gathered}
$$

In (18) and (19), PEP is inversely proportional to the product of link average SNR values of the channels. Since there are 4 statistically independent channels a diversity order of 4 is achieved when $d_{1}$ and $d_{2}$ is not equal to zero.

Case-2: only one user successfully decodes the information $\left(\varepsilon_{1}=0, \varepsilon_{2}=1\right.$ or $\varepsilon_{1}=1, \varepsilon_{2}=0$ ). In this 
case only one portion of the code word achieves the MRC. Suppose Relay-1 decode the information correctly and Relay- 2 can't i.e $\varepsilon_{1}=1, \varepsilon_{2}=0$. So $N_{1}$ portion of the coded bits get MRC and $N_{2}$ portion of coded bits is received only from source. In this case the conditional PEP is,

$$
\begin{aligned}
& P\left(d \mid \gamma_{1 a}, \gamma_{1 b}, \gamma_{2 a}\right) \\
& =Q\left(\sqrt{2 d_{1} \gamma_{1 a}+2 d_{1} \gamma_{1 b}+2 d_{2} \gamma_{2 a}}\right)
\end{aligned}
$$

From (15) and (16) we can find the exact unconditional PEP and Upper bond of PEP as,

$$
\begin{aligned}
& P(d)= \\
& \frac{1}{\pi} \int_{0}^{\pi / 2}\left(1+\frac{d_{1} \overline{\gamma_{1 a}}}{\sin ^{2} \theta}\right)^{-1}\left(1+\frac{d_{1} \overline{\gamma_{1 b}}}{\sin ^{2} \theta}\right)^{-1}\left(1+\frac{d_{2} \overline{\gamma_{2 a}}}{\sin ^{2} \theta}\right)^{-1} \\
& P(d) \leq \frac{1}{2}\left(\frac{1}{1+d_{1} \overline{\gamma_{1 a}}}\right)\left(\frac{1}{1+d_{1} \overline{\gamma_{1 b}}}\right)\left(\frac{1}{1+d_{2} \overline{\gamma_{2 a}}}\right)
\end{aligned}
$$

Here diversity of order 3 is achieved. Only one portion of the coded bits achieves spatial diversity and the other portion is only transmitted by source. Case-3: both users fail to decode information bits $\left(\varepsilon_{1}=\varepsilon_{2}=0\right)$. In this case the destination receives both $N_{1}$ and $N_{2}$ from the source and both the relay restrain from transmission in the second phase. The conditional PEP for this ase is

$$
P\left(d \mid \gamma_{1 a}, \gamma_{1 b}, \gamma_{2 a}\right)=Q\left(\sqrt{2 d_{1} \gamma_{1 a}+2 d_{2} \gamma_{2 a}}\right)
$$

The unconditional exact PEP and the upper bound is

$$
\begin{gathered}
P(d)=\frac{1}{\pi} \int_{0}^{\pi / 2}\left(1+\frac{d_{1} \overline{\gamma_{1 a}}}{\sin ^{2} \theta}\right)^{-1}\left(1+\frac{d_{2} \overline{\gamma_{2 a}}}{\sin ^{2} \theta}\right)^{-1} d \theta \\
P(d) \leq \frac{1}{2}\left(\frac{1}{1+d_{1} \overline{\gamma_{1 a}}}\right)\left(\frac{1}{1+d_{2} \overline{\gamma_{2 a}}}\right)
\end{gathered}
$$

There is no spatial diversity for this case. A diversity of order 2 is achieved because the source transmits two portions of coded bits in two coherent phases of independent fading coefficients (we termed this case as single user time diversity).

\subsubsection{Fast fading case}

In fast fading the coefficients for each channel are i.i.d. over time period and antenna. The conditional PEP can be denoted as

$$
P(d \mid \gamma)=Q(\sqrt{2 d \gamma(n)})
$$

The instantaneous received SNR for the nth bit of code word $N_{1}$ and $N_{2}$ is given by,

$$
\begin{aligned}
\gamma_{1}(n) & =\frac{P_{s}\left|q_{1, S D}(n)\right|^{2}}{N_{0}}+\varepsilon_{1} \frac{P_{R}\left|q_{1 D}(n)\right|^{2}}{N_{0}} \\
& =\gamma_{1 a}(n)+\varepsilon_{1} \gamma_{1 b}(n) \\
\gamma_{2}(n) & =\frac{P_{s}\left|q_{2, S D}(n)\right|^{2}}{N_{0}}+\varepsilon_{1} \frac{P_{R}\left|q_{2 D}(n)\right|^{2}}{N_{0}} \\
& =\gamma_{2 a}(n)+\varepsilon_{2} \gamma_{2 b}(n)
\end{aligned}
$$

For a total block size $N=N_{1}+N_{2}$ equation (26) can rewrite as

$$
\begin{gathered}
P\left(d \mid \gamma_{1 a}, \gamma_{1 b}, \gamma_{2 a}, \gamma_{2 b}\right)= \\
Q\left(\sqrt{2 \sum_{n=\eta_{1}}\left\{\gamma_{1 a}(n)+\varepsilon_{1} \gamma_{k b}(n)\right\}+2 \sum_{n=\eta_{2}}\left\{\gamma_{2 a}(n)+\varepsilon_{2} \gamma_{2 b}(n)\right\}}\right)
\end{gathered}
$$

where, the set $\eta_{1}$ has the cardinalities of $\mathrm{d} 1$ and $\eta_{2}$ has the cardinalities of $d_{2}$. Using the same approach of [13] as slow fading we can find the expression of unconditional PEP as,

$$
\begin{aligned}
& P(d)= \\
& \frac{1}{\pi} \int_{0}^{\pi / 2} \prod_{n=\eta 1}\left[\int_{0}^{\infty} \exp \left(-\frac{\gamma_{1 a}(n)}{\sin ^{2} \theta}\right) P\left(\gamma_{1 a}(n)\right) d \gamma_{1 a}(n)\right] \\
& \times \prod_{n=\eta 1}\left[\int_{0}^{\infty} \exp \left(-\frac{\varepsilon_{1} \gamma_{1 b}(n)}{\sin ^{2} \theta}\right) P\left(\gamma_{1 b}(n)\right) d \gamma_{1 b}(n)\right] \\
& \times \prod_{n=\eta 2}\left[\int_{0}^{\infty} \exp \left(-\frac{\gamma_{2 a}(n)}{\sin ^{2} \theta}\right) P\left(\gamma_{2 a}(n)\right) d \gamma_{2 a}(n)\right] \\
& \times \prod_{n=\eta 2}\left[\int_{0}^{\infty} \exp \left(-\frac{\varepsilon_{2} \gamma_{2 b}(n)}{\sin ^{2} \theta}\right) P\left(\gamma_{2 b}(n)\right) d \gamma_{2 b}(n)\right] d \theta
\end{aligned}
$$

The exact solution of (30) for Rayleigh distribution of all fading coefficients can be given as [13], 


$$
\begin{aligned}
& P(d) \\
& =\frac{1}{\pi} \int_{0}^{\pi / 2}\left(1+\frac{d_{1} \overline{\gamma_{1 a}}}{\sin ^{2} \theta}\right)^{-d_{1}}\left(1+\varepsilon_{1} \frac{d_{1} \overline{\gamma_{1 b}}}{\sin ^{2} \theta}\right)^{-d_{1}} \\
& \left(1+\frac{d_{2} \overline{\gamma_{2 a}}}{\sin ^{2} \theta}\right)^{-d_{2}}\left(1+\varepsilon_{2} \frac{d_{2} \overline{\gamma_{2 b}}}{\sin ^{2} \theta}\right)^{-d_{2}} d \theta
\end{aligned}
$$

where, $\bar{\gamma}$ represent the average SNR. The upper bound of PEP is,

$$
\begin{aligned}
P(d) \leq & \frac{1}{2}\left(\frac{1}{1+d_{1} \overline{\gamma_{1 a}}}\right)^{d_{1}}\left(\frac{1}{1+d_{1} \varepsilon_{1} \overline{\gamma_{1 b}}}\right)^{d_{1}} \\
& \times\left(\frac{1}{1+d_{2} \overline{\gamma_{2 a}}}\right)^{d_{2}}\left(\frac{1}{1+d_{2} \varepsilon_{2} \overline{\gamma_{2 b}}}\right)^{d_{2}}
\end{aligned}
$$

Similar to the slow fading case we have to consider the 3 different cases of cooperation. We can easily write the exact solution for PEP and the upper bound by replacing the values of $\varepsilon_{1}$ and $\varepsilon_{2}$ in equation (31) and (32) as slow fading case. In case-1 $\left(\varepsilon_{1}=\mathcal{E}_{2}=1\right)$ : A diversity order of $2 \mathrm{~d}$ is achieved, case-2 $\left(\varepsilon_{1}=0, \varepsilon_{2}=1\right.$ or $\left.\varepsilon_{1}=1, \varepsilon_{2}=0\right)$ achieved a diversity of order $\left(\mathrm{d}+d_{1}\right)$ or $\left(\mathrm{d}+d_{2}\right)$ and in case- $3\left(\varepsilon_{1}=\varepsilon_{2}=0\right)$ diversity of order $\mathrm{d}$ is achieved. In [4], shows that diversity order of $d$ is also achievable in direct transmission case i.e. time diversity does not give any extra benefit in fast fading case. Our proposed protocol doubles the diversity order when two relays cooperate.

\subsection{Bit and Frame Error Rate Analysis}

For a code having rate $1 / \mathrm{n}$ with Viterbi decoder the error event probability for case $C=\{1,2,3\}$ can easily be calculated using the well known Viterbi's upper bound as [15]

$$
P_{E}(C) \leq \sum_{d=f r e e}^{\infty} a_{d} P(d \mid \gamma, C)
$$

where, dfree is the free distance of the code and $a_{d}$ is the number of error evens with Hamming distance d. Conditional Frame Error Rate (FER) probability in terms of error event probabilities of a decoded block of length $B$ can be calculated using the approach of [16] as,

$$
\begin{aligned}
P_{F E R}(C) & \leq 1-\left(1-P_{E}(\gamma, C)\right)^{B} \\
& \leq B P_{E}(\gamma, C) \\
& \leq B \cdot \sum_{d=d_{j r e t}}^{\infty} a_{d} P(d \mid \gamma, C)
\end{aligned}
$$

The conditional Bit Error Rate (BER) of case C $=\{1,2,3,4\}$ is also can be calculated using Viterbi's upper bound for bit error probability [15] as.

$$
P_{B E R}(\gamma, C) \leq \sum_{d=d_{f, e \epsilon}}^{\infty} c_{d} P(d \mid \gamma, C)
$$

where, $c_{d}$ is the information error weight on all paths with $d \geq d_{\text {free }}$.

BER and FER for individual cases can calculate by replacing the value of $P(d)$ in equation (34) from (18), (21) and (24) for case-1, 2 and 3 respectively. The so called distance spectra $a_{d}$ and $c_{d}$ which should be as small as possible, depend only on the code. Therefore we have to choose a suitable code that optimizes the performance of our protocol. In this paper we testrict out analysis only on diversity gain; therefore, we omit the code selection policy to optimize the coding gain. Interested reader can follow the code selection policy for coded cooperation discussed in [5]. To calculate end-to-end BER and FER we need to calculate the probability of individual cases $C=\{1$, $2,3\}$ as,

$$
\begin{gathered}
\operatorname{Pr}(C=1)=\left(1-P_{F E R}^{R 1}\right)\left(1-P_{F E R}^{R 2}\right) \\
\operatorname{Pr}(C=2)=P_{F E R}^{R 1}\left(1-P_{F E R}^{R 2}\right)\left(1-P_{F E R}^{R 1}\right) P_{F E R}^{R 2} \\
\operatorname{Pr}(C=3)=P_{F E R}^{R 1} P_{F E R}^{R 2}
\end{gathered}
$$

where, $P_{F E R}^{R 1}$ and $P_{F E R}^{R 1}$ is FER at relay-1 and relay-2 which can be calculated using equation (34) considering only one fading path with code corresponding to $N_{1}$ bits.

Finally, the over all unconditional BER and FER is the average over the three possible cases of 
transmission and given by

$$
\begin{aligned}
& P_{F E R}=\sum_{i=1}^{3} P_{F E R}(C) \cdot \operatorname{Pr}(C=i) \\
& P_{B E R}=\sum_{i=1}^{3} P_{B E R}(C) \cdot \operatorname{Pr}(C=i)
\end{aligned}
$$

\section{Numerical and Simulation Results}

Simple BPSK modulation is considered for simplicity. We use $1 / 4$ rate convolutional code with polynomial generator (23 3527 33) and 16-bit CRC code with generator polynomial 15935 (hexadecimal number). The puncturing is done for $50 \%$ cooperation where puncturing period $\mathrm{P}=4$ and

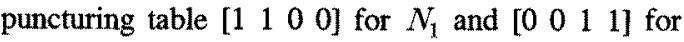
$N_{2}$. We consider source block size $=256$ bits. AWGN noise is modeled as zero mean complex random variable with variance $N_{0} / 2$ per dimension. For simplicity assume $N_{0}=1$. This assumption doesn't affect the results because $N_{0}$ is already included in our SNR definition in equation (6). All the fading coefficients are modeled as un-correlated samples of Rayleigh fading, where the values are generated by zero mean complex random variable with variance 0.5 per dimension. In real cases, the signal components from relay nodes are dominant due to network topology when path loss is taken into account. In fact, cooperative communication can achieve both diversity gain and path loss reduction (like, multi-hop communication). In this paper we consider a symmetrical network i.e., the variance of source to destination and relays to destination fading coefficient are same. If we consider the relays are close to the destination than the source then the performance of our protocol will further improve due to the path loss reduction. To isolate path loss reduction and diversity gain we consider the symmetrical network. We assume the fading coefficient for user to destination is constant during the transmission of every phase for slow fading, but independent in different phases. We also assume that the perfect channel state information is available at the receiver so that coherent detection is possible.

Fig. 2 shows the comparison of BER of the proposed system with direct transmission and a single user time diversity based transmission in slow fading. For direct transmission we consider the fading is constant over both code word ( $N_{1}$ and $N_{2}$ ). In case of single user time diversity case we assume the user transmits $N_{1}$ and $N_{2}$ bit in independent coherent channel time, i.e. fading is independent for two phases. This case is same as conventional coded cooperation proposed in $[3,4]$; because, two frame experience independent fading path. In order to make fair comparison we restrict the equal total power transmission and data rate from all nodes. We consider Ps=Pt in case of direct transmission, $P_{s_{1}}=P_{S_{2}}=P_{t}$ in case of single user with time diversity (or coded cooperation) and $P_{s_{1}}$ $=P_{s_{2}}=\operatorname{Pr}_{1}=\operatorname{Pr}_{2}=P_{t} / 2$ for the proposed two relay cooperative protocol. Simulation result shows that our proposal outperforms the direct transmission and conventional coded cooperation protocol.

In fig. 2 we also verify our simulation results with analytical BER computation. The solid lines are the simulation (S)results and the doted lines represent the approximation found by numerical upper bound (UB). We truncate the summations of equation (35) to the first 7 terms of $d$ and $c_{d}$. We use MATLAB function 'distspec' to evaluate the

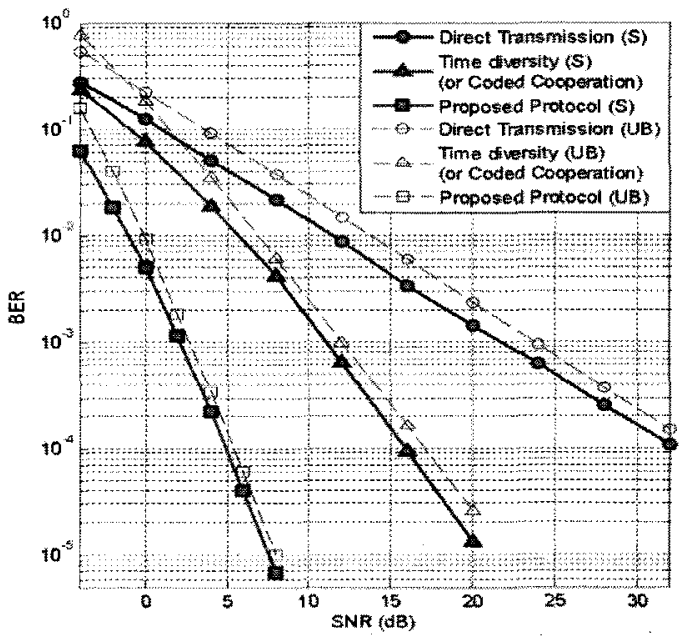

Fig, 2. BER performance comparison. 
values of $\mathrm{d}$ and $c_{d}$. In all cases the analytical approximations agree very well with the simulations. The upper bound truncation is tight at high SNR but it is relatively loose at low SNR, because we consider limit after averaging technique explained in [16]. Stricter upper bound can be get using the limit after averaging technique but this approach required a 4 fold numerical integration to evaluate $P(d)$ for case 1 . The simulation results also agree with the mathematical diversity analysis. Single user time diversity over two time slot offer a diversity of order 2 (same as conventional coded cooperation) and the new protocol offer a diversity of order 4. Similar results for FER are also possible.

The spatial diversity gain of the proposed system is sensitive to the source to relay channel condition. In good source to relay channel condition the probability of detecting the information at relay is high and the system get the maximum benefit from the relaying. In fig. 3 we investigate the various sources to relay channel conditions. We consider a symmetric network i.e., similar source to relay-1 and relay-2 channel and relays to destination channel statistics.

To observe the end-to-end performance we normalize the distance between any node (source or

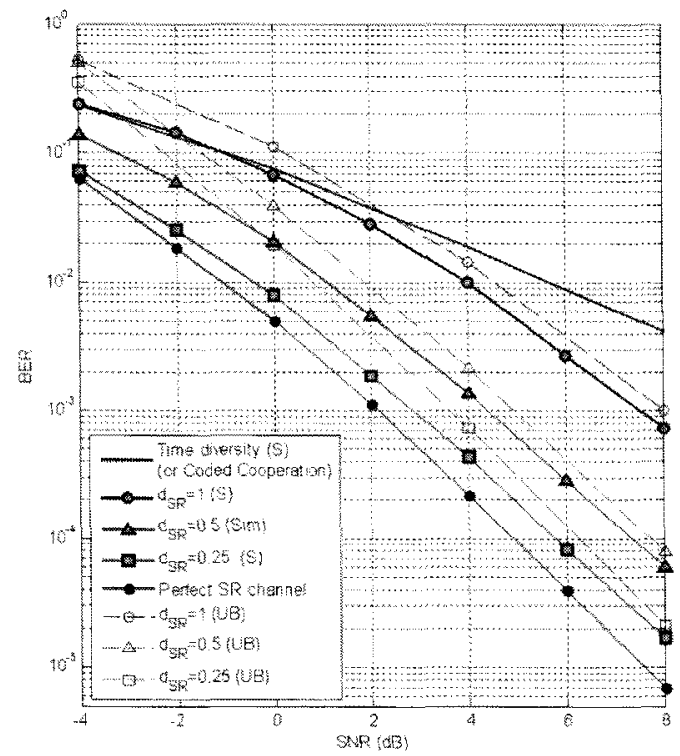

Fig. 3. Investigate the effect of various sources to relay channel conditions on end-to-end BER performance. relay) to destination to 1 . To isolate path loss reduction from diversity gain we assume signal components at destination from source and relays experiences same path loss. Now, to see the effect of source to relay channel condition on cooperation we consider the normalized distance between source and relays $1,0.5$ and 0.25 . To realize the effects of this normalized distance we can model the variance of channel fading coefficient between node $i$ and $j$ as a function of distance between two nodes $i$ and j $\sigma_{i j}=l_{i j}^{-a b}$ where ais so called path loss exponent that vary from 2 to 6 on the basis of channel environment and $I_{i j}$ is the distance between node $\mathrm{i}$ and $j$. In simulation we consider $a=3$.

Fig. 3 shows that the end-to-end performance of our proposal is better than ideal single user time diversity even for distances between source to relays $1=1$. The performance improves as the distance between source and relay decreases. The simulation and upper bound confirms that the end-to-end BER is very close to the ideal source to relay channel condition when $1=0.25$. In all cases the simulation results of end-to-end BER agree well with analytical upper bound.

\section{Conclusions}

We investigate the coded cooperation system in wireless network where a single active user is supported by two idle users in relay mode. In all kind of wireless network this is a common scenario that a number of idle users are available to assist the active users in relay mode and we exploit this idle node to get spatial diversity. Our work focuses on the issue that the users benefited from the diversity along with coding gain as channel coding is the basic part of all kind of wireless communication. So the performance of the system is better than the system having equal data rate and equal total power transmit. We also take the advantage of time diversity to increase the diversity order. The proposed protocol can be applied in any type of wireless network like cellular base system, wireless sensor network ad-hoc network etc. 


\section{References}

[1] A. Hedayat, T. E. Hunter, A. Nosratinia, "Cooperative Communication in Wireless Networks" IEEE communication Magazine, Vol.42, No.10, pp.68-73, October-04.

[2] T. E. Hunter and A. Nosratinia, "Cooperative diversity through coding," in Proc. IEEE International Symposium on Information Theory (ISIT), Laussane, Switzerland, p.220, July 2002.

(3) T. E. Hunter and A. Nosratinia. "Performance analysis of coded cooperation diversity." IEEE international conference on communications (ICC). Anchorage, AK, p.2688, May 2006.

(4) M. Janani, A. Hedayat, T. E. Hunter, A. Nosratinia, "Coded cooperation in wireless communications: Space-time transmission and iterative decoding," IEEE Trans. Signal Processing, Vol.52, No.2, pp.362-371, Feb. 2004.

[5] A. Stefanov and E. Erkip, "Cooperative Coding for Wireless Networks" IEEE transactions on communications, Vol.52, No.9, Sep. 2004.

(6) J. Hagenauer, "Rate-Compatible Punctured Convolutional Codes (RCPC Codes) and Their Applications," IEEE Trans. Commun., Vol.36, No.4, pp.389-400, April 1988.

(7) Ho Van Khuong, Hyung-Yun Kong, and Dong-Un Lee, "User-Cooperation and Cyclic Coding in Wireless Sensor Networks", JKIPS (Journal of Korea Information Processing Society) Trans. on Communications, Vol.13-C, No.3, pp.317-322, June 2006.

[8] Ho Van Khuong and Hyung-Yun Kong, "Performance Analysis of Cooperative Communications Protocol using Sum-Product Algorithm for Wireless Relay Networks", Proceedings of The 8th IEEE International Conference on Advanced Communication Technology,Vol.3, pp.2168-2173, Phoenix Park, Korea, 20-22 Feb. 2006.

[9] Ho Van Khuong and Hyung-Yun Kong, "Performance Analysis of Cooperative Transmission under Strict Power Constraints",
IEICE Trans. on Communications, Vol.E89-B, No.3, pp.1007-1011, March 2006.

[10] Paul A. Anghel, Geert Leus and Mostafa Kaveh, "On the performance of distributed space-time coding systems with one and two non-regenerative relays" IEEE Trans. On wireless Communications, Vol.5, Issue 3, pp.682-692, March 2006.

[11] Paul A. Anghel, and Mostafa Kaveh, "Exact symbol error probability of a cooperative network in Rayleigh fading environment". IEEE Trans. On wireless Communications, Vol.3, Issue 5, pp.1416-1421, Sept. 2004.

〔12〕 Gordon L. Stuber, "Principles of mobile communication, second edition, Kluwer Academic publisher.

(13] M. K. Simon and M. S. Alouini "Digital communication over generalized fading channel" Jhon Wiley and Sons 2000.

(14) J. W Craig "a New simple and exact result for calculating the probability of error for 2-dimentional signal constellations", in Proc. IEEE MILCOM, McLean, VA, pp.571-575, October 1991,

[15] Viterbi and J. K. Omura, Principles of Digital Communcation and Coding, New York: McGraw-Hill, 1979.

[16] E. Malkamaki and H. Leib, "Evaluating the performance of convolutional codes over block fading channels," IEEE Trans. Inform. Theory, Vol.45, No.5, pp.1643-646, July 1999.

[17] Todd E. Hunter, Shahab Sanayei, Aria Nosratinia," Outage Analysis Of Coded Cooperation", IEEE Transactions On Information Theory, Vol.52, No.2, February 2006.

[18] Asaduzzaman, K. Hyung-Yun, and S. Kim Nae, "Coded Cooperation Diversity in Wireless Relay Network," in International Conference on Information and Communication Technology, ICICT '07, pp.123-126, 2007. 


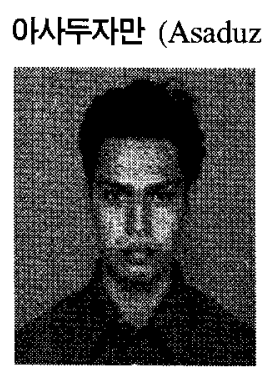

Rgular Member Asaduzzaman received the $\mathrm{B}$. Sc. Engineering Degree in ele-ctrical and electronics engineering from Chittagong University of Engineering and Technology, Bangladesh, in 2001. From 2001 to 2005 he was a faculty member of the same University. He is currently working toward the Ph.D. degree in the Department of Electrical Engineering, niversity of Ulsan, Korea. His major research interests include wireless communication systems with emphasis on cooperative communications and MIMO systems, wireless sensor networks, modulation and coding techniques.

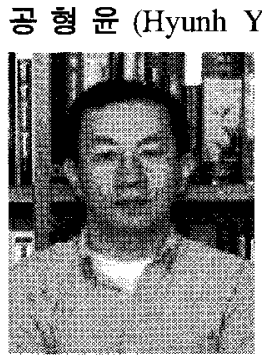

engineering from the New York Institute of Technology, New York, in 1989. Since 1996, he has been employed by LG Electronics Co., Ltd., in the multimedia research lab. Currently he is a professor in Electrical Engineering at University of Ulsan, Korea. His research area includes channel coding detection and estimation, cooperative communications, cognitive radio and sensor networks. I am a member of KICS, IEEK, KIPS, IEEE and IEICE. He performs several projects supported by NRF (National Reserch foundation of Korea), Hyundai heavy industries Co. Ltd. 